\title{
Novel Features of Classical Electrodynamics and Their Connection to the Elementary Charge, Energy Density of Vacuum and Heisenberg's Uncertainty Principle-Review and Consolidation
}

\author{
Vernon Cooray ${ }^{*}$, Gerald Cooray ${ }^{2}$ \\ ${ }^{1}$ Department of Engineering Sciences, Uppsala University, Uppsala, Sweden \\ ${ }^{2}$ Karolinska Institute, Stockholm, Sweden \\ Email: *vernon.cooray@angstrom.uu.se, gerald.cooray@ki.se
}

How to cite this paper: Cooray, V. and Cooray, G. (2019) Novel Features of Classical Electrodynamics and Their Connection to the Elementary Charge, Energy Density of Vacuum and Heisenberg's Uncertainty Principle-Review and Consolidation. Journal of Modern Physics, 10, 74-90.

https://doi.org/10.4236/jmp.2019.101007

Received: December 6, 2018

Accepted: January 26, 2019

Published: January 29, 2019

Copyright $\odot 2019$ by author(s) and Scientific Research Publishing Inc. This work is licensed under the Creative Commons Attribution International License (CC BY 4.0).

http://creativecommons.org/licenses/by/4.0/

\begin{abstract}
The paper provides a review and conciliation of the results pertinent to the energy and action associated with electromagnetic radiation obtained using classical electrodynamics and published in several journal papers. The results presented in those papers are based on three systems that generate electromagnetic radiation, namely, frequency domain antennas, time domain antennas and decelerating (or accelerating) charged elementary particles. In the case of radiation generated by a frequency domain antenna, the energy dissipated as radiation within half a period, $U$, satisfies the order of magnitude inequality $U \geq h v \rightarrow q \geq e$ where $q$ is the magnitude of the oscillating charge in the antenna, $e$ is the elementary charge, $v$ is the frequency and $h$ is the Planck constant. In the case of transient radiation fields generated by time domain antennas or the radiation emitted by decelerating (or accelerating) charged elementary particles, the energy dissipated by the system as radiation satisfies the order of magnitude inequality $U \tau_{r} \geq h / 4 \pi \rightarrow q \geq e$ where $U$ is the energy dissipated as radiation by the system, $\tau_{r}$ is the duration of the energy emission and $q$ is either the charge in the current pulse in the case of the time domain antenna or the charge of the elementary particle giving rise to the radiation. These results are derived while adhering strictly to the principles of classical electrodynamics alone. These results were interpreted in different papers in different ways using different assumptions. In this paper, we provide a unified interpretation of the results, and combining these results with two simple quantum mechanical concepts, expression for the
\end{abstract}


elementary charge as a function of other natural constants and the energy density of vacuum is derived. The expressions predict the elementary charge to an accuracy higher than about $1 \%$.

\section{Keywords}

Classical Electrodynamics, Electromagnetic Radiation, Electron, Elementary Charge, Vacuum Energy, Dark Energy, Hubble Radius, Heisenberg's Uncertainty Principle

\section{Introduction}

In several recent publications, Cooray and Cooray [1] [2] [3] [4] [5] investigated the features of electromagnetic radiation generated by antennas working in both frequency and time domain when the radiating charge in the antenna is reduced to the elementary charge. These authors studied the same problem from a different angle by studying the electromagnetic radiation generated by a decelerating charged elementary particle when it strikes an impenetrable perfectly conducting boundary [6]. These studies revealed the existence of several remarkable features associated with the electromagnetic radiation fields as predicted by classical electrodynamics. Even though these studies were conducted independent of each other, the derived results had several similar features. The goal of this paper is to place all investigations in the same framework, review the results, and provide unified conclusions concerning these predictions of classical electrodynamics. Thus, the concepts used here are already presented in the six papers mentioned above. The mathematical expressions related to the energy, momentum, and action of the radiation fields are identical to those presented previously in the papers mentioned above. For this reason, final equations are obtained directly from the respected papers without presenting the derivations. However, some of the definitions of the parameters used here are slightly different from the ones used in those papers and this was done to achieve uniformity in the method of analysis utilized in different studies. Moreover, in the case of the transient anten$\mathrm{na}$, the problem analyzed in the previous paper is a dipole antenna whereas here, a monopole located over a perfectly conducting ground plane is examined.

In Section 2 we will show the results obtained purely based on classical electrodynamics. In Section 3 and 4 it is shown how the results obtained in Section 2 can be combined with the known concepts of quantum mechanics to extract expressions for the elementary charge in terms of other natural constants and the energy density of vacuum. Section 5 presents a summary of the results and conclusions.

\section{Analysis}

\subsection{Frequency Domain Antenna}

As the radiating system we will consider an antenna of length $L$ located over a 
perfectly conducting ground plane. The perfectly conducting plane coincides with the $x-y$ plane with $z=0$. The antenna is located along the $z$-axis and is fed by a sinusoidal current from the ground end (i.e. $\mathrm{z}=0$ ). The material to be given below is obtained directly from reference [2].

The power generated by the antenna takes place in bursts of duration $T / 2$, where $T$ is the period of oscillation. Under ideal conditions when all the losses associated with the current propagation along the antenna can be neglected, the median value of the energy radiated within a single burst of duration $T / 2$ is given by the equation

$$
U_{\text {med }}=\frac{q_{0}^{2} \pi v}{4 \varepsilon_{0} c}\{\gamma+\ln (4 \pi L / \lambda)\}
$$

In Equation (1), $\gamma$ is Euler's constant, which is equal to 0.5772, $q_{0}$ is the magnitude of the oscillating charge, $\lambda$ is the wavelength, and $v$ is the frequency of oscillation. For a given charge $q_{0}$, the median energy increases with increasing length of the antenna and decreasing wavelength. Let us consider the limits of $L$ and $\lambda$. The smallest possible value of the wavelength that one can plug into the equation is in the order of the antenna radius. In the analysis to follow, we assume that the smallest possible radius of an antenna that can exist in nature is equal to the Bohr radius $a_{0}$. We also presume that the largest possible value of the antenna length that one can have in nature is equal to the ultimate radius of the universe that is in causal contact with the observer. This radius is estimated as follows. Consider an observer in an expanding universe. The objects in this universe which are not bound to the observer by any other forces such as gravitational, electromagnetic or nuclear are receding with respect to the observer with a certain speed which increases with the distance from the observer to the object. Consequently, as the distance between the observer and the object increases, there is a certain distance where the objects recede from the observer with the speed of light. This distance is called the "Hubble radius". The space located beyond the Hubble radius expands faster than the speed of light and the observer cannot receive any information from events happening beyond the Hubble radius. Thus, the maximum spatial distance available for the observer within the space that is in causal contact with him is the Hubble radius. The Hubble radius varies with the age of the universe and in the present epoch of the universe the Hubble radius increases with the age of the universe. However, the presence of vacuum energy will provide an upper asymptotic limit to the Hubble radius, a steady state value. Thus, in our universe the maximum length of an antenna that an observer can achieve is limited by this steady state value of the Hubble radius. Let us denote this steady state value of the Hubble radius by $R_{\infty}$. Using this as the ultimate limit of the antenna length, the upper limit of the median energy dissipated within a single power burst of duration $T / 2$ for a given charge is given by,

$$
U_{\max }=\frac{q_{0}^{2} \pi v}{4 \varepsilon_{0} c}\left\{\gamma+\ln \left(4 \pi R_{\infty} / a_{0}\right)\right\}
$$


Consider a photon of frequency $v$. The energy associated with such a photon is given by $h v$, where $h$ is the Planck constant. The charge that corresponds to a median energy equivalent to a single photon is given by

$$
q_{0}= \pm \sqrt{\frac{4 \varepsilon_{0} h c}{\pi\left\{\gamma+\ln \left(4 \pi R_{\infty} / a_{0}\right)\right\}}}
$$

Now, the steady state or the ultimate value of the Hubble radius is given by

$$
R_{\infty}=c^{2} \sqrt{\frac{3}{8 \pi G \rho_{\Lambda}}}
$$

In the above equation, $G$ is the gravitational constant and $\rho_{\Lambda}$ is the energy density of the vacuum. The current estimate of the vacuum energy density is about $6 \times 10^{-10} \mathrm{~J} / \mathrm{m}^{3}$ [7]. Substituting this in the above equation we find that the final value of the Hubble radius i.e. $R_{\infty}$ is about $1.55 \times 10^{26} \mathrm{~m}$. Substituting the expression for $R_{\infty}$ from Equation (4) in Equation (3), we obtain

$$
q_{0}= \pm \sqrt{\pi\left\{\gamma+\ln \left[\frac{4 \pi c^{2}}{a_{0}} \sqrt{\left.\frac{3}{8 \pi G \rho_{\Lambda}}\right]}\right]\right\}}
$$

If we substitute numerical values to the parameters in the above equation we find that $q_{0}= \pm 1.603 \times 10^{-19}$ which is equal to the elementary charge to an accuracy of $0.1 \%$. This indicates that in an oscillatory antenna radiating at its maximum capacity which is limited only by the natural dimensions, an oscillating charge with magnitude equal to the elementary charge will generate, in a single burst of radiation, an energy equivalent to a single photon. If the length of the antenna is smaller or the radius of the antenna is larger than the natural limits, a charge larger than an elementary charge is needed to radiate an equivalent amount of energy in a single burst. Moreover, since the antenna losses will reduce the amount of energy dissipation for a given charge, if one includes the antenna losses in the analysis, a larger charge will be necessary to compensate for the losses. The results can be summarized by the following inequality: $U \geq h v \rightarrow q \geq e$. In this inequality, $U$ is the energy associated with a single burst of radiation, $h$ is the Planck constant, $q$ is the magnitude of the oscillating charge, and $e$ is the elementary charge. Note that $q_{0}$ (i.e. the smallest value of $q$ ) is not exactly equal to $e$. Thus, this inequality has to be treated as an order of magnitude relationship. Observe that the reverse of this inequality is not valid because $q \geq e$ does not lead to $U \geq h v$.

\subsection{Time Domain Antenna}

Consider a long and vertical conductor located over a perfectly conducting ground plane. As in the previous case the perfectly conducting plane coincides with the plane $x-y$ with $z=0$. A current is injected into this antenna from the ground end $(z=0)$. Recall that, as before, we are considering the ideal case without any losses. That is, we neglect all the losses such as thermal, dispersive 
and radiative. In this case the pulse propagates along the antenna without attenuation and dispersion. We consider the case in which the current pulse gets absorbed when it reaches the end of the antenna.

The current waveform propagating along the antenna is assumed to be a Gaussian pulse of the form $\mathrm{e}^{-t^{2} / 2 \sigma^{2}}$. The duration of this pulse is denoted by $\tau$. In order to make the analysis general, we define a non-dimensional parameter $\eta$ as follows:

$$
\eta=\tau /(L / c)
$$

Note that $\eta$ denotes the ratio between the duration of the current and the time of travel of the current along the antenna. Following the same procedure used in references [1] [3] and [4], the energy dissipated by the antenna can be written as

$$
U=\frac{q^{2}}{8 \pi^{3 / 2} \sigma \varepsilon_{0} c} \ln \left(\frac{2}{\eta}\right)
$$

Let us now consider the action associated with this energy burst. The action is defined as the product of the dissipated energy and the time over which the energy is dissipated. The temporal variation of power associated with the radiation burst varies as $\mathrm{e}^{-t^{2} / 2 \sigma_{r}^{2}}$ where $\sigma_{r}=\sigma / \sqrt{2}$. We define the duration of the energy burst $\tau_{r}$, as the time interval within which $95 \%$ of the energy is dissipated. With this definition we obtain $\tau_{r} \approx 4 \sigma_{r}=2^{3 / 2} \sigma$. Obviously, one can use slightly different definitions for the duration of the energy burst [see references [1] [3] and [4]] but such choices will not change the order of magnitude of the results to be obtained. Thus, the action associated with this radiation burst is given by

$$
A=\frac{\tau_{r} q^{2}}{8 \pi^{3 / 2} \sigma \varepsilon_{0} c} \ln \left(\frac{2}{\eta}\right)
$$

Substituting for $\tau_{r}$ and after simplification we obtain

$$
A=\frac{2^{3 / 2} q^{2}}{8 \pi^{3 / 2} \varepsilon_{0} c} \ln \left(\frac{2}{\eta}\right)
$$

After substituting for $\eta$ the action can be written as

$$
A=\frac{2^{3 / 2} q^{2}}{8 \pi^{3 / 2} \varepsilon_{0} c} \ln \left(\frac{2 L}{\tau c}\right)
$$

As per this equation, the action associated with a given charge $q$ increases with increasing $L$ and with decreasing $\tau c$. Let us consider the natural limits of these parameters. Now, the radiation field as given earlier is valid when $\tau c$ is on the order of the radius of the conductor or larger. This is the case because if this condition is not satisfied the radiation fields generated from different parts of the cross section will interfere destructively reducing the amplitude of the radiation field. Thus, the maximum value of the radiation, and hence the action is obtained, when $\tau c \approx a$, where $a$ is the radius of the conductor. For a given radius, the maximum value of the action is then given by 


$$
A=\frac{2^{3 / 2} q^{2}}{8 \pi^{3 / 2} \varepsilon_{0} c} \ln \left(\frac{2 L}{a}\right)
$$

Using the same arguments as in the case of frequency domain analysis, the maximum value of the length of the conductor that one can have is equal to $R_{\infty}$ and the smallest radius is equal to the Bohr radius. Thus, the maximum action associated with a given charge is

$$
A_{m}=\frac{2^{3 / 2} q^{2}}{8 \pi^{3 / 2} \varepsilon_{0} c} \ln \left(\frac{2 R_{\infty}}{a_{0}}\right)
$$

Now, the natural unit that is being used to measure the action is $h / 2 \pi$ or $h / 4 \pi$. The charge necessary to make the action equal to $h / 4 \pi$ is given by

$$
q_{0}= \pm \sqrt{\frac{h \pi^{1 / 2} \varepsilon_{0} c}{2^{1 / 2}}\left[1 / \ln \left(\frac{2 R_{\infty}}{a_{0}}\right)\right]}
$$

Substituting numerical values for the parameters in the above equation we find $q_{0}= \pm 1.61 \times 10^{-19} \mathrm{C}$. This shows that as the charge decreases the action decreases and when the charge reaches the elementary charge the action becomes comparable to $h / 4 \pi$. Since Equation (12) gives the maximum action possible in nature for a given charge, one can summarize the results by the following inequality: $A \geq h / 4 \pi \rightarrow q \geq e$ where $A$ is the action associated with a burst of radiation generated by the antenna and $q$ is the charge associated with the current. Observe that the reverse of this inequality is not valid. That is, $q \geq e$ does not lead to $A \geq h / 4 \pi$. Note that this result is based purely on classical electrodynamics without burrowing any features from quantum electrodynamics.

\subsection{Radiation Fields Generated by a Decelerating Charged Elementary Particle or Transition Radiation}

In the previous sections we have analyzed the radiation fields generated by transmitting antennas, both in the frequency domain and time domain, when their dimensions are pushed to limits allowed by nature. In this section, we will summarize the results obtained in reference [6] for the radiation fields generated when a fast-moving charged elementary particle is incident on a perfectly conducting ground plane. As before the perfectly conducting plane is located along an $x-y$ plane with $z=0$. A charged elementary particle moves in the direction of negative $z$-axis with uniform velocity $v$ and is incident on the ground plane. The deceleration of the particle during this encounter gives rise to a pulse of electromagnetic radiation. If one treats the elementary particle as a point particle the radiation goes to infinity. Thus, to calculate the radiation field, we will treat the charged elementary particle as a charge distribution and consider the movement of the particle as a propagation of a current pulse. The temporal variation of the current pulse is assumed to be Gaussian. The energy dissipated during the encounter between the charged particle and the perfect conductor is given by (with $\beta=v / c)$; 


$$
U=\frac{q^{2} \beta^{2}}{16 \pi^{3 / 2} \varepsilon_{0} c \sigma}\left[\frac{1+\beta^{2}}{\beta^{3}} \ln \left(\frac{1+\beta}{1-\beta}\right)-\frac{2}{\beta^{2}}\right]
$$

As in the previous case, the temporal variation of the power emission is given by $\mathrm{e}^{-t^{2} / \sigma^{2}}$, and as before the duration of the energy emission $\tau_{r}=2^{3 / 2} \sigma$. Thus, the action associated with this radiation energy burst is given by

$$
A=\tau_{r} U=\frac{q^{2} \beta^{2} 2^{3 / 2}}{16 \pi^{3 / 2} \varepsilon_{0} c}\left[\frac{\left(1+\beta^{2}\right)}{\beta^{3}} \ln \left(\frac{1+\beta}{1-\beta}\right)-\frac{2}{\beta^{2}}\right]
$$

In our study, we are interested in the values of $\beta$ where $(1-\beta) \ll 1$, i.e. $\beta \approx 1$. Under these conditions, the action reduces to

$$
A=\frac{q^{2} 2^{3 / 2}}{8 \pi^{3 / 2} \varepsilon_{0} c}\left[\ln \left(\frac{2}{1-\beta}\right)-1\right]
$$

Observe that the action reaches infinity when $\beta$ goes to unity; i.e. when the speed of the particle reaches the speed of light. Due to relativistic change of mass of the particle its speed can never become equal to the speed of light. However, in principle, there is no restriction for the speed of the particle to reach values which are infinitesimally close to the speed of light. Assume that the particle is moving with speed of light along the z-direction. Since, the speed of light is the maximum ever possible speed of the charged particle, this indicates that the speed of the particle in any direction along the $\mathrm{x}-\mathrm{y}$ plane is equal to zero. However, this contradicts the Heisenberg's position-momentum uncertainty principle. Since the particle is confined inside the Hubble sphere, according to Heisenberg's uncertainty principle the speed of the particle in any given direction could not be equal to zero. Perivolaropoulos analyzed this problem in reference [8], and according to the results there is a minimum momentum associated with the particle in any given direction, and the speed $v_{\min }$ of the particle associated with this minimum momentum is given by [8]

$$
v_{\min }=\frac{3 \sqrt{3}}{8} \frac{h}{\pi} \frac{1}{m_{e} R_{\infty}}
$$

Thus, the maximum speed of the particle in any given direction in space is limited to a value $v_{\max }$ given by

$$
v_{\max } \approx c-v_{\min }
$$

This will limit the minimum value of $(1-\beta),(1-\beta)_{\min }$, to

$$
(1-\beta)_{\min }=\frac{3 \sqrt{3}}{8 \pi} \frac{h}{m R_{\infty} c}
$$

In the above equation $m$ is the mass of the charged elementary particle. Substituting this in Equation (16) we obtain the maximum value of the action associated with any given charge as

$$
A_{m}=\frac{q^{2} 2^{3 / 2}}{8 \pi^{3 / 2} \varepsilon_{0} c}\left[\ln \left(\frac{16 \pi m R_{\infty} c}{3 \sqrt{3} h}\right)-1\right]
$$


The charge necessary for this action to be equal to $h / 4 \pi$ is given by

$$
q_{0}= \pm \sqrt{\frac{h \pi^{1 / 2} \varepsilon_{0} c}{2^{1 / 2}\left[\ln \left(\frac{16 \pi m R_{\infty} c}{3 \sqrt{3} h}\right)-1\right]}}
$$

Consider a charged elementary particle having the mass identical to an electron. Replacing the mass $m$ in Equation (21) by the mass of the electron $m_{e}$ and re-writing the equation in terms of well-known atomic parameters we obtain

$$
q_{0}= \pm \sqrt{\frac{h \pi^{1 / 2} \varepsilon_{0} c}{2^{1 / 2}\left[\ln \left(\frac{8 R_{\infty}}{3 \sqrt{3} \alpha a_{0}}\right)-1\right]}}
$$

In the above equation, $\alpha$ and $a_{0}$ are the fine structure constant and the Bohr radius respectively. Equation (22) gives the charge necessary in an elementary particle having a mass equal to that of an electron so that the action becomes comparable to $h / 4 \pi$. Substituting numerical values for the parameters in the above equation, we find $q_{0} \approx \pm 1.58 \times 10^{-19} \mathrm{C}$. This shows that when the charge of the elementary particle having a mass equal to that of an electron reaches the elementary charge, the action becomes comparable to $h / 4 \pi$. Since the Equation (20) gives the maximum action possible in nature for a given charge of magnitude $q$, the results can be summarized by the following inequality: $A \geq h / 4 \pi \rightarrow q \geq e$. Observe that this relationship is valid for an elementary particle having the mass of an electron. Also note the condition $A=h / 4 \pi \rightarrow q \approx e$ is satisfied when the elementary charged particle travels with the maximum possible speed allowed by nature for a particle located inside the Hubble sphere. Moreover, observe again that the reverse of this inequality is not valid. That is, $q \geq e$ does not lead to $A \geq h / 4 \pi$.

The results presented above are obtained for an elementary particle having a mass equal to that of an electron. The other charged elementary particles are Quarks, muons and taus. However, quarks are not free and confined inside the nucleus. Both muons and taus decay rapidly to electrons. Muons decay to electrons within about $2.2 \times 10^{-6}$ seconds and taus decay to electrons within about $10^{-13}$ seconds. However, the relationship is approximately satisfied also by both muons and taus, thanks to the fact that the mass of the particle appears inside the logarithmic term.

\section{Discussion}

\subsection{Frequency Domain Antenna}

In the analysis, we have assumed the length and radius of the radiating antenna have the extreme limits allowed by nature. Let us refer to such an antenna as the "ultimate antenna". It is important to note that if the antenna length is decreased or the antenna radius is increased from these extreme values, the magnitude of 
the radiation will decrease. In other words, to reach the same level of radiation as the extreme antenna, more charge is necessary in the radiating antenna. The same is true if we include the losses associated with the current propagation. The effect of these losses is to reduce the magnitude of the radiation fields, and more charge is necessary in the radiating system to reach the same level of radiated energy. This means that the condition $U \geq h v \rightarrow q \geq e$ is valid for an antenna of any length or radius and even when losses are included. In other words, it is a universal condition. The condition $U \geq h v \rightarrow q=e$ is satisfied by the ultimate antenna and the condition $U \geq h v \rightarrow q \geq e$ is satisfied by any other antenna. Observe that this relationship is obtained using classical electrodynamics alone. It is important to point out that this relationship does not prove that the minimum charge that can oscillate in the antenna is the elementary charge. It only shows that if, and only if, $U \geq h v$ then $q \geq e$. If we wish to prove that the smallest possible oscillating charge in the antenna is the elementary charge, we have to show that the condition $U \geq h v$ is satisfied by the electromagnetic radiation. Let us consider this point in more detail.

In the inequality, $U$ is the energy dissipated within the time $T / 2$. According to quantum mechanical interpretation electromagnetic radiation consists of photons. If any electromagnetic energy is dissipated within the time duration $T / 2$, then at least one or more photons should be released by the radiator during this time interval. Since the energy of a photon cannot be smaller than $h v$, the energy released during the time period $T / 2$ cannot be smaller than $h v$. In other words, the quantum mechanical nature of electromagnetic radiation gives rise to inequality $U \geq h v$. This shows that the condition $q \geq e$ is a consequence of the quantum nature of the electromagnetic fields.

Equation (5) is derived using pure classical electrodynamics and it indicates $q_{0}$ is almost equal to the elementary charge. If we assume that $q_{0}$ is exactly equal to the elementary charge, then, Equation (5) can be written as

$$
e=\sqrt{\pi\left\{\gamma+\ln \left[\frac{4 \pi c^{2}}{a_{0}} \sqrt{\left.\frac{3}{8 \pi G \rho_{\Lambda}}\right]}\right]\right\}}
$$

Using the above expression for the elementary charge one can express the fine structure constant $\alpha$ as

$$
\alpha=\frac{2}{\pi\left\{\gamma+\ln \left[\frac{4 \pi R_{\infty}}{a_{0}}\right]\right\}}
$$

In terms of the vacuum energy density the fine structure constant is given by

$$
\alpha=\frac{2}{\pi\left\{\gamma+\ln \left[\frac{4 \pi c^{2}}{a_{0}} \sqrt{\left.\frac{3}{8 \pi G \rho_{\Lambda}}\right]}\right]\right\}}
$$

Note that even though Equations (5) and (23) are almost equivalent they are 
based on very different assumptions. In Equation (5) $q_{0}$ is the charge necessary in the ultimate antenna so that the median energy dissipated over half a period, $U$, is equal to $h v$. This is a result based purely on classical electrodynamics. The value of $q_{0}$ is almost equal to the elementary charge (to an accuracy less than $1 \%$ ) and we assume that $q_{0}=e$. However, the condition $q_{0}=e$ is valid only if the value of $U$ does not go below $h v$. However, classical electrodynamics does not place any restrictions on the value $U$ and accordingly, it can take any value larger than zero. Indeed, one has to appeal to quantum mechanics to show that the minimum value of $U$ cannot be less than $h v$. The statement $q_{0}=e$ is based, therefore, on quantum nature of the electromagnetic radiation. Thus, Equation (23) is a result of the fact that electromagnetic radiation consists of photons.

Equation (23) can be rewritten while making $\rho_{\Lambda}$ the subject as

$$
\rho_{\Lambda}=\frac{3}{8 \pi G\left\{\frac{a_{0}}{4 \pi c^{2}} \exp \left[\frac{4 \varepsilon_{0} h c}{e^{2} \pi}-\gamma\right]\right\}^{2}}
$$

Equation (26) is an expression for the energy density of vacuum in terms of well-known physical constants. If we substitute numerical values to these constants, we find $\rho_{\Lambda}=4 \times 10^{-10} \mathrm{~J} / \mathrm{m}^{3}$ which is close to the measured value $6 \times 10^{-10} \mathrm{~J} / \mathrm{m}^{3}$. Indeed, this is the value we need in Equation (5) to make $q_{0}=e$. Equation (26) can be considered as an alternative expression for the vacuum energy density.

In a glance, it is difficult to understand how the charge of an electron and the size of the steady state (or the ultimate) Hubble radius, as indicated by Equations (5) and (23), are connected. We believe that the relationship between the size of the universe and the elementary charge is a secondary relationship. As per Equations (5) and (23), the true primary connection is probably associated with the energy density of the vacuum and the elementary charge.

\subsection{Time Domain Antenna}

The results presented here are based on the work presented in references [1] [3] and [4]. However, there are a few differences in the definition of the parameters. For example, here, the duration of the radiation field is defined by analyzing the temporal variation of the power of the radiation field whereas in references [1] [3] [4] and [6] the duration is defined using the current waveform. This difference in the definition of the duration of the energy burst had caused a few percent change in the calculated value of $q_{o}$. One more difference is the fact that in references [1] [3] and [4] the radiating antenna consists of a dipole whereas here a monopole antenna over a perfectly conducting ground is used. The aim here was to evade the problem of two current waveforms taking part in the radiation.

As in the case of the frequency domain antenna, a reduction in length of the antenna or an increase in the radius of the antenna will lead to a reduction in the energy dissipation. That means, to generate the same level of radiation as the ul- 
timate antenna, the charge associated with the current has to increase. Thus, the relationship $A \geq h / 4 \pi \rightarrow q \geq e$ is satisfied by all time-domain radiating antennas. The relationship $A=h / 4 \pi \rightarrow q=e$ is valid for the ultimate antenna and the relationship $A>h / 4 \pi \rightarrow q>e$ is valid for any other antenna. Since the losses will decrease the amount of radiation generated by a given antenna, an antenna with losses requires a larger charge to maintain the same level of energy emission. Thus, the relationship $A>h / 4 \pi \rightarrow q>e$ is valid for all antennas even when the losses are included. One can indeed claim that the condition is universally satisfied by time domain antennas. It is vital to stress here again that these results are based purely on classical electrodynamics.

As in the previous case, one cannot conclude that the smallest free charge that can radiate is equal to the elementary charge by solely depending on the results obtained from classical electrodynamic. Actually, the quantization of the charge is observed about 30 years after the development of classical electrodynamics, and it is not a requirement in that theory. If we are interested in proving from the above relationship that the smallest free charge available in nature is the elementary charge, it is necessary to prove that the condition $U \tau_{r} \geq h / 2 \pi$ is satisfied by the radiated energy. Let us now investigate whether one can prove the condition $U \tau_{r} \geq h / 2 \pi$ is satisfied by the radiation emitted by the time domain antenna.

Consider an experiment in which the radiation emitted by a time domain antenna is utilized to estimate the location of the antenna. Now, for values of $1 / \eta$ which are larger than about $10^{5}$, which indeed is the case under consideration, the z-component of the momentum transported by radiation is given by $U / c$ [5]. Note that due to symmetry, the net momentum has only a $\mathrm{z}$ component. Let $\Delta U$ be the uncertainty in $U$. Then the uncertainty in the z-component of the momentum transported by the radiation is $\Delta U / c$. As the radiation is emitted, an equal but opposite momentum is transferred to the antenna. Consequently, the uncertainty in the z-component of the momentum of the antenna, $\Delta p_{z}$, will also be $\Delta U / c$. Recall also that the duration of the power emitted by the antenna is $\tau_{r}$ and under the conditions mentioned earlier this power is dissipated mainly along the $\mathrm{z}$-direction. For this reason, the antenna can be located in the z-direction only to an accuracy of about $c \tau_{r}$, i.e. $\Delta z=c \tau_{r}$. These uncertainties must satisfy the position momentum uncertainty principle, i.e. $\Delta z \Delta p_{z}=h / 4 \pi$. Substituting for $\Delta z$ and $\Delta p_{z}$ in the latter we obtain $\tau_{r} \Delta U=h / 4 \pi$. Now, when the charge responsible for the radiation is on the order of the elementary charge, the uncertainty in the energy is comparable to the energy itself. This is the case, because the elementary charge is the smallest possible charge that can radiate. As a result, when the charge associated with the current is comparable to the elementary charge, the Heisenberg's uncertainty principle leads to $\tau_{r} U=h / 4 \pi$. When the charge associated with the radiation is larger than the elementary charge, the uncertainty in the energy becomes larger than the minimum uncertainty given above, and one can write $\tau_{r} U \geq h / 2 \pi$. Based on this 
analysis, one can conclude that the condition $q \geq e$ is a consequence of the Heisenberg's uncertainty principle (see also reference [5]).

In deriving the above relationship, one had to make several assumptions concerning the definition of the duration of the radiation burst and the shape of the current pulse. For these reasons the inequality derived here can be considered as an order of magnitude approximation. It is important to point out that the results presented here are based on a Gaussian current pulse. Since we ultimately reduce the charge to an electron, the Gaussian pulse could be the most reasonable approximation to represent the charge associated with an electron. However, one can represent the current with other waveforms such as finite step, exponential decay etc. As pointed out in references [1] even with other current waveforms the order of magnitude of the relationship still holds. Note also that the smallest value of $q$ for a give radiation level is obtained for the largest value of the Hubble radius. As the Hubble radius decreases, the value of $q$ increases and hence the above inequality is not disturbed even when using a Hubble radius smaller than the steady state one (we will take up this point later).

In the analysis the current pulse is assumed to propagate with the speed of light along the antenna. It is vital to point out that what is propagating with the speed of light is the current pulse and not the individual charges themselves.

\subsection{Transition Radiation}

While estimating the maximum speed that a particle can have when confined to the Hubble radius we had to appeal to results obtained using quantum mechanics (i.e., Heisenberg's uncertainty principle). Other than that, the derivation is based purely on classical electrodynamics. Following are the other significant points to be considered:

In the case of transition radiation, we have concluded that if $A \geq h / 4 \pi$ then $q \geq e$. As in the case of the transient antenna, one can show independently that the constraint $A \geq h / 4 \pi$ is satisfied by the transition radiation. The existence of such a constraint in nature can be understood by appealing again to the Heisenberg's uncertainty principle. Consider the charged elementary particle that will give rise to the transition radiation. The particle is moving along the $\mathrm{z}$-axis with a well-defined speed and hence momentum. Due to Heisenberg's uncertainty principle its location is largely uncertain. At a particular time, the particle will interact with the perfectly conducting plane and gives rise to the transition radiation. Let us consider a hypothetical or "Gedanken" experiment in which the transition radiation itself is used to locate the position of the particle. Since the particle is moving very close to the speed of light, most of the radiation is emitted along the z-axis and the $\mathrm{z}$-component of the momentum associated with the radiation is given by $U / c$. Due to the removal of energy as radiation from the particle, its momentum in the direction of $\mathrm{z}$-axis will become uncertain by an amount equal to $U / c$. That is, the z-momentum uncertainty, $\Delta p_{z}$, of the particle will be about $U / c$. Let us now consider the accuracy with which the loca- 
tion of the particle can be estimated. Since the transition radiation is generated over a time interval equal to $\tau_{r}$, the z-location of the particle as estimated using this radiation will be uncertain by an amount equal to $v \tau_{r}$. That is, $\Delta z=v \tau_{r}$. These two uncertainties should satisfy the position-momentum uncertainty principle, and therefore

$$
\tau_{r} v \frac{U}{c} \geq \frac{h}{4 \pi}
$$

For values of $v$ close to the speed of light, it reduces to the equation

$$
\tau_{r} U \geq \frac{h}{4 \pi}
$$

To obtain the uncertainty in the location of the particle, one can also appeal to the following argument. If one uses electromagnetic radiation of wavelength $\lambda$ to locate a particle, it can be located only to an accuracy on the order of $\lambda$. Transition radiation, however, is a time domain pulse and its effective wavelength is about $\tau_{r} c$. Thus, the uncertainty in the position of the particle in the $\mathrm{z}$-direction as estimated from the transition radiation is about $\tau_{r} c$. Use of this uncertainty in position and the uncertainty in the z-momentum estimated earlier in the uncertainty principle will also lead to Equation (28). In the previous section we showed that "if" the condition $A \geq h / 4 \pi$ is valid then $q \geq e$. Equation (28) indicates that the condition $A \geq h / 4 \pi$ indeed is satisfied by radiated energy. This makes it possible for us to derive an expression for the magnitude of the smallest free charge available in nature in terms of other physical parameters. For example, since the condition $A \geq h / 4 \pi$ is satisfied by the emitted radiation, we can consider Equation (22) as an expression for the magnitude of the smallest free charge available in nature. This equation predicts the elementary charge to an accuracy close to $1 \%$.

\section{Effects of Including the Time Dependent Hubble Radius}

In our analysis, we assumed that the analysis of radiation from antennas and transition radiation is conducted in an epoch of the universe where the Hubble radius remains constant. Based on this assumption and appealing to two quantum mechanical concepts, we have derived several expressions for the elementary charge and the predictions of these equations agree within $1 \%$ with the measured value. We believe that this is the correct assumption to be made because in this epoch we will have a truly constant "Hubble constant" and Hubble radius. Moreover, such an assumption links the elementary charge to the energy density of vacuum which is a local parameter of the fabric of space in which the electrons are located. However, it is of interest to find out the consequences on the results if this assumption is relaxed. Assume that the radiation is analyzed in an epoch where the Hubble radius is still increasing with time. In this case, the expressions obtained for the magnitude of the elementary charge can be written as (with $R(t)$ denoting the Hubble radius at time $t$ ) 


$$
\begin{gathered}
q_{0}=\sqrt{\frac{4 \varepsilon_{0} h c}{\pi\left\{\gamma+\ln \left(4 \pi R(t) / a_{0}\right)\right\}}} \\
q_{0} \approx \sqrt{\frac{\varepsilon_{0} \pi^{1 / 2} c h}{2^{1 / 2} \ln \left(\frac{2 R(t)}{a_{0}}\right)}} \\
q_{0} \approx \sqrt{\frac{\varepsilon_{0} \pi^{1 / 2} c h}{2^{1 / 2}\left[\frac{8 R(t)}{\ln \left(\frac{8 \sqrt{3} \alpha a_{0}}{3}\right)}-1\right]}}
\end{gathered}
$$

Note that we have used the approximately equal sign (i.e. $\approx)$ in Equations (30) and (31) because in deriving these two equations we had to appeal to the Heisenberg's uncertainty principle which provides an order of magnitude estimation of relevant quantities. Using the Friedmann equation, one can calculate how the Hubble radius varies as a function of time [9] [10]. Figure 1(a) presents the results of this calculation. The smallest age of the universe used in the calculation is 0.1 million years.

The Hubble radius increases with time and reaches a constant value decided by the vacuum energy density. If the values of $q_{0}$ in all the three examples are estimated using the Hubble radius corresponding to each epoch, the resulting values of $q_{0}$ as a function of the age of the universe for the three examples considered in this paper are depicted in Figure $1(\mathrm{~b})$. Even if we consider the variation of $q_{0}$ as a function of the age of the universe, one can still treat the relationships $U \geq h v \rightarrow q \geq e$ and $\tau_{r} U \geq h / 4 \pi \rightarrow q \geq e$ as the order of magnitude estimates. Further, the smallest value of $q$ for a give radiation level is obtained

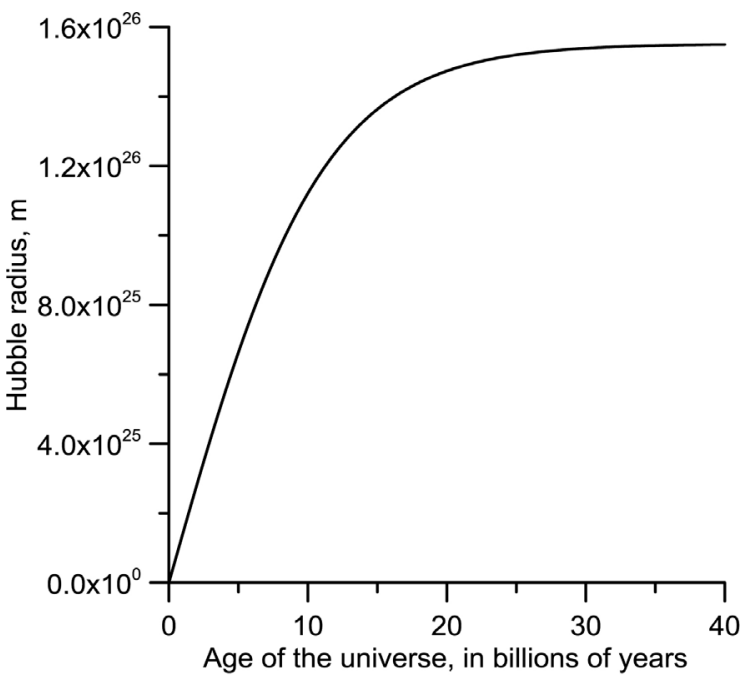

(a)

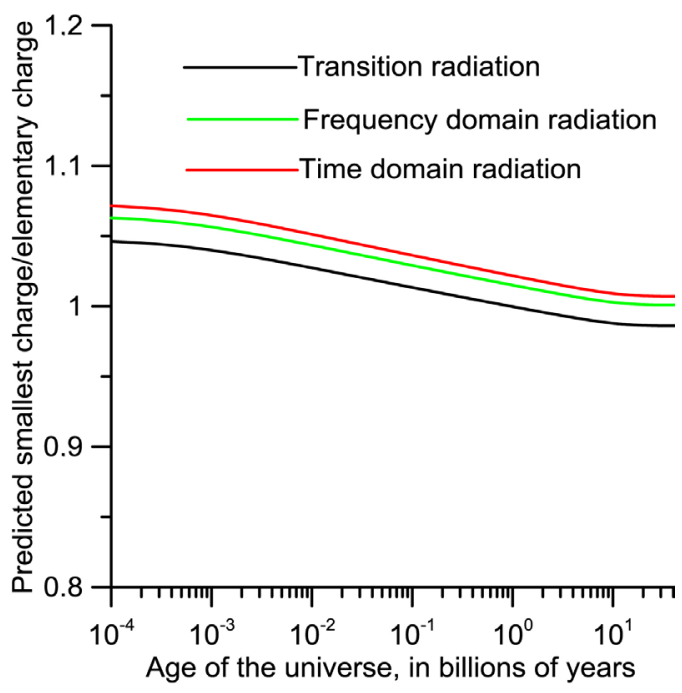

(b)

Figure 1. (a) The variation of Hubble radius with the age of the universe. (b) The variation of the estimated minimum charge, $q_{0}$, for the three cases considered when the Hubble radius corresponding to the age of the universe is utilized. Note that the charge in Figure $1(b)$ is given as a fraction of the elementary charge. Also note that the current age of the universe is about 13.8 billion years. 
for the largest value of the Hubble radius. As the Hubble radius decreases the value of $q$ increases and for this reason the above inequalities are not disturbed when using a Hubble radius smaller than the steady state one. Of course, one can make the predicted charge and the fine structure constant to be independent of the age of the universe or the size of the Hubble radius by treating the speed of light as a variable inversely proportional to the Hubble radius while the impedance of free space remains constant. That is $c R(t)=$ constant and $\sqrt{\mu_{0} / \varepsilon_{0}}=$ constant. In this case, the speed of light will have a high value close to the beginning of the universe and decreases as the universe ages. We believe, however, that the correct procedure is to use the steady state Hubble radius in the calculation because this is the largest spatial radius in the universe that an observer can have within which the events taking place are in causal contact. In this case we predict a direct relationship between the elementary charge and the energy density of vacuum. This connection appears logical to us because the radiating electrons are located in a space and time controlled by the vacuum energy which in turn controls the ultimate size of the universe.

\section{Summary and Conclusions}

At present, the radius of the universe as given by the Hubble radius increases with time. Thanks to the vacuum energy this radius reaches a steady value as the time increases. This is the maximum value of the Hubble radius ever possible in the current universe. Let us denote this steady and maximum Hubble radius by $R_{\infty}$. The radiating systems to be considered here are located within $R_{\infty}$. The main results obtained for these radiating systems can be summarized as follows:

1) Consider an ideal antenna located over a perfectly conducting ground plane containing a sinusoidally oscillating current. Increase the length of the antenna to $R_{\infty}$ and decrease the radius of the antenna to the Bohr radius. Now, reduce the magnitude of the oscillating charge in the antenna to the elementary charge. Then the energy generated within half a period will be reduced to $h v$. Based on this result, one can show that for any antenna with oscillating currents the radiated energy satisfies the inequality $U \geq h v \rightarrow q \geq e$ where $U$ is the energy dissipated as radiation within half a period of the antenna and $q$ is the oscillating charge in the antenna. It is also possible to utilize the results to extract an expression that connects the energy density of the vacuum to the elementary charge.

2) Consider an ideal antenna located over a perfectly conducting ground plane through which a current pulse propagates with speed of light in free space. Increase the length of the antenna to $R_{\infty}$ and decrease the radius of the antenna to the Bohr radius. Let $U$ be the energy radiated by the antenna and $\tau_{r}$ is the time over which this radiation is generated. Now, reduce the magnitude of the current so the charge associated with it becomes equal to the elementary charge. Then the action associated with the radiation $U \tau_{r}$ reduces to $h / 4 \pi$. Based on this result, one can show that for any antenna with transient currents the ra- 
diated energy satisfies the inequality $U \tau_{r} \geq h / 4 \pi \rightarrow q \geq e$ where $q$ is the charge in the transient current.

3) Consider a charged elementary particle that is moving with the maximum possible speed in the universe at a time when the Hubble radius has reached its steady value $R_{\infty}$. The particle strikes a perfectly conducting ground plane and the deceleration of the charged particle during this encounter gives rise to transition radiation. Let $U$ be the energy dissipated in the transition radiation and $\tau_{r}$ is the time over which this radiation is generated. It can be proved that when the charged particle is an electron the action associated with the radiation $U \tau_{r}$ reduces to $h / 4 \pi$. Based on this result, one can show that the transition radiation generated by an accelerating or decelerating charged elementary particle satisfies the order of magnitude inequality $U \tau_{r} \geq h / 4 \pi \rightarrow q \geq e$ where $q$ is the charge of the elementary particle. The inequality is satisfied to a high degree of accuracy by an electron. As far as an order of magnitude relationship, it is also satisfied by other elementary charged particles, namely muons and taus.

The inequalities derived in this paper are valid even if one considers any other epoch of the universe where the Hubble radius is still increasing and it is less than the steady state value. Based on the results, one can conclude that all radiating systems that generate frequency domain radiation satisfy the condition $U \geq h v \rightarrow q \geq e$ and all radiating systems that generate transient electromagnetic radiation satisfy the condition $U \tau_{r} \geq h / 4 \pi \rightarrow q \geq e$. These results, obtained purely using classical electrodynamics, when combined with basic quantum mechanical concepts show that the elementary charge and the vacuum energy are intimately connected.

\section{Acknowledgements}

Authors appreciate the suggestions, support and encouragement given by Prof. Farhad Rchidi, Prof. Marcos Rubinstein, Prof. Carlo Mazzetti and Prof. Yoshihiro Baba during the development of the ideas presented in this paper.

\section{Conflicts of Interest}

The authors declare no conflicts of interest regarding the publication of this paper.

\section{References}

[1] Cooray, V. and Cooray, G. (2016) Atmosphere, 7, 64. ttps://doi.org/10.3390/atmos7050064

[2] Cooray, V. and Cooray, G. (2018) Journal of Electromagnetic Analysis and Application, 10, 77-87. https://doi.org/10.4236/jemaa.2018.105006

[3] Cooray, V. and Cooray, G. (2017) Atmosphere, 8, 46. https://doi.org/10.3390/atmos8030046

[4] Cooray, V. and Cooray, G. (2017) Journal of Electromagnetic Analysis and Application, 9, 167-182. https://doi.org/10.4236/jemaa.2017.911015

[5] Cooray, V. and Cooray, G. (2016) Atmosphere, 7, 151. 
https://doi.org/10.3390/atmos7110151

[6] Cooray, V. and Cooray, G. (2017) Natural Science, 9, 219-230.

https://doi.org/10.4236/ns.2017.97022

[7] Komatsu, E., et al. (2011) Astrophysical Journal, 192, 18.

https://doi.org/10.1088/0067-0049/192/2/18

[8] Perivolaropoulos, L. (2017) Physical Review D, 95, Article ID: 103523.

[9] Weinberg, S. (2008) Cosmology. Oxford University Press, Oxford.

[10] Cooray, V., Cooray, G. and Rachidi, F. (2017) Journal of Modern Physics, 8, 1979-1987. https://doi.org/10.4236/jmp.2017.812119 